\section{Next-generation dendritic cell vaccines}

\section{By Lev Osherovich, Senior Writer}

Vaccine makers have known for years that delivering immunogenic antigens to dendritic cells can elicit the strong $\mathrm{T}$ cell-based immune response that is key to combating viral and bacterial infections and cancer. But complexities of vaccine formulation and dosing have made delivering the antigens to dendritic cells in vivo a challenge.

Now, American researchers have devised two methods to maximize the uptake and presentation of antigens by dendritic cells (DCs) and have demonstrated potent cellular immunity in mice and monkeys vaccinated using the techniques.

DCs are innate immune system components that sponge up extracellular debris, then process and present foreign antigens to cells of the acquired immune system.

Loading up and activating DCs is the idea behind Dendreon Corp.'s Provenge sipuleucel-T, an autologous cell therapy marketed for metastatic, castrate-resistant prostate cancer. But Provenge requires isolating and culturing DCs from the patient, activating them ex vivo and reintroducing them back into the patient's circulation.

A multi-campus team led by Robert Seder, senior investigator and chief of cellular immunology at the National Institute of Allergy and Infectious Diseases, hopes to cut out the ex vivo step.

The team reported two alternative strategies for maximizing DC activation in vivo in papers published in the Proceedings of the National Academy of Sciences and The Journal of Clinical Investigation. ${ }^{1,2}$ The first strategy involves targeting antigens directly to DCs using a $\mathrm{mAb}$ and then following up with a viral booster shot. The second strategy is to conjugate antigens to a small molecule adjuvant and deliver this complex as a large aggregate, making it more likely than soluble antigens to attract the attention of DCs.

Using the new methods, "you can actually target certain dendritic cells that are better for eliciting a helper $\mathrm{T}$ cell response or a cytotoxic T cell response" than DCs isolated in cell culture, said Seder.

"The concept here is to catch the attention of the immune system to get antigens to be more effectively processed by dendritic cells," said David Urdal, EVP and CSO at Dendreon.

\section{One-two punch}

In the PNAS study, Seder's team collaborated with co-senior author Ralph Steinman, professor and senior physician at The Rockefeller University, to test a two-step immunization strategy aimed at maximizing DC response to a viral antigen.

The team vaccinated macaques with a fusion of HIV gag p24 protein and a $\mathrm{mAb}$ that recognized a DC surface protein called lymphocyte antigen 75 (LY75; DEC-205). Previous work by Steinman's team showed that DEC-205 is a marker of a subset of innate immune cells that are effective at launching $\mathrm{T}$ cell responses. ${ }^{3}$

The injection was accompanied by an adjuvant that activates the antigen-sensing toll-like receptor 3 (TLR3) on the surface of DCs.

Monkeys receiving the vaccine-adjuvant combination had longer-lasting antigen-specific cytotoxic T lymphocyte (CTL) and type I interferon responses, as well as longer $\mathrm{T}$ helper cell activity, than controls that received adjuvant plus antigen without a DC-targeting antibody.

Seder's team obtained further improvements in both cytotoxic and T helper cell activity by giving vaccinated monkeys a booster shot of New York vaccinia virus engineered to express a variety of HIV antigens.

Previous immunological studies by his own team and others suggested that if "you want to develop a $T$ cell response, you'll probably need to use a couple of different vaccine platforms," said Seder. "Here we used heterologous prime boost, in which we use two different types of vaccine to get a better response" than is possible with a single vaccine.

Last year, Steinman and colleagues at Rockefeller started a Phase I trial of the targeted antigen-adjuvant combination described in the PNAS study in HIV-positive patients.

\section{Aggregate advantage}

In the JCI study, Seder's team hypothesized that antigens made to look like the large complexes of debris routinely encountered by DCs would be better at eliciting immune responses than naked antigen.

The researchers conjugated a model protein antigen-a bacterial ovalbumin - to resiquimod, a small molecule agonist of TLR7 and TLR8. Like TLR3, TLR7 and TLR8 are found on the surface of DCs, where they recognize foreign antigens.

Celldex Therapeutics Inc. has rights from $3 \mathrm{M}$ Co. to use resiquimod as an adjuvant in DC vaccines.

Mice vaccinated with the aggregated antigen-adjuvant complex had a more vigorous $\mathrm{T}$ cell and type I interferon response than controls receiving co-injected but unconjugated antigen and adjuvant or a soluble version of the antigen-adjuvant conjugate.

Seder thinks the conjugated antigen-adjuvant aggregate may be a potent activator of DCs because those cells seem to take it up more readily than soluble protein.

He said combining the aggregation formulation technique with the heterologous prime-boost strategy in the first study could lead to an even greater $\mathrm{T}$ cell response, but that possibility remains to be tested. 
Figure 1. Two dendritic cell vaccines. Flynn et al. and Kastenmüller et al. outline contrasting strategies for triggering robust cellular immunity in vivo.

Flynn et al. immunized monkeys with an antibody against the dendritic cell (DC) protein lymphocyte antigen 75 (LY75; DEC205) fused to a model antigen, HIV gag p24 [a(1)] plus poly-ICLC adjuvant [a(2)]. PolyICLC is a synthetic double-stranded RNA formulated together with polylysine and carboxymethylcellulose that induces activation of toll-like receptor 3 (TLR3). In vivo, the primary vaccine and adjuvant was taken up by DCs [a(3)], which were further stimulated by a boosting vaccination of an attenuated New York vaccinia virus engineered to express HIV proteins [a(4)]. The resulting activated DCs elicited a robust cytotoxic $T$ lymphocyte (CTL) and T helper cell response against HIV compared with that seen in controls receiving conventional vaccination formulations and regimens [c].

Kastenmüller et al. vaccinated mice with an aggregate of a conjugate made up of the model antigen ovalbumin and the small molecule vaccine adjuvant resiquimod $[\mathbf{b}(1)]$, which engaged TLR7 and TLR8 [b(2)]. After a booster shot of the same formulation [b(3)], activated DCs led to a strong CTL and T helper cell response [c] against ovalbumin in vaccinated mice compared with in controls given soluble antigen.

Oncovir Inc. has Hiltonol poly-ICLC in Phase I and Phase II testing as an adjuvant to immunotherapy against HIV and a range of cancers. Celldex Therapeutics Inc. has rights from $3 \mathbf{M}$ Co. to use resiquimod as an adjuvant in DC vaccines.
Targeted strategy

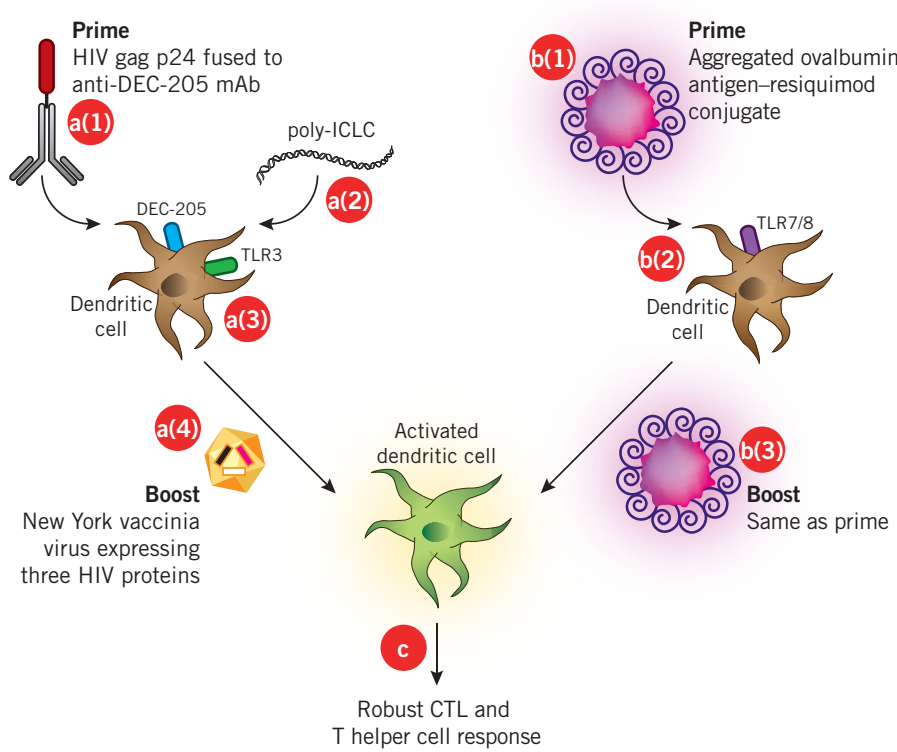

"For maximal protection, [aggregation] is half the puzzle-the other half is the viral boost," said Seder.

His next step is to test variations on the two strategies in animal models of malaria and tuberculosis (TB), two indications in which $\mathrm{T}$ cell responses are critical for immunity.

Rockefeller has patented the DC-targeting technology used in the PNAS study. Seder did not file for patents on the findings in the JCI study.

\section{Interferon key}

The two studies present proof of concept for contrasting strategies for maximizing DC activation by vaccines (see Figure 1, "Two dendritic cell vaccines").

In principle, either method could be used to generate immunity against a range of infectious diseases and tumor antigens. But which method is best suited for which indications, and how the methods measure up against more conventional DC vaccination strategies already in the clinic, remains to be seen.

Franck Barrat, director of drug discovery at Dynavax Technologies Corp., said the methods were "very complementary. These papers educate people about some of the key rules of vaccine design-conjugation of the adjuvant and targeting the antigen to the dendritic cells."
"These papers educate people about some of the key rules of vaccine design-conjugation of the adjuvant and targeting the antigen to the dendritic cells."

-Franck Barrat,

Dynavax Technologies Inc. Corp.
Barrat said the monkey studies are especially compelling, as differences in adjuvant efficacy between mice and primates have led vaccine makers astray in the past.

"By going to the extra effort in primates, they are really doing strong work," he said.

According to Barrat, the take-home message of the studies is that vaccine makers should aim to elicit a strong and lasting type I interferon response by DCs, which Barrat suspects to be the cause of the vigorous $\mathrm{T}$ cell responses seen in Seder's studies.

"Type I interferon is the place to go, whether it's through the targeted method or the aggregation method," said Barrat.

Dynavax's Heplisav (V720), a formulation of hepatitis B surface antigen (HBsAg) given with a TLR9 agonist, is in Phase III testing to prevent $\mathrm{HBV}$ infection in chronic kidney disease (CKD) patients.

Urdal said Seder's techniques might be worth testing in cancer immunotherapy, though he noted that building up immunity against tumor antigens is a different game than creating a protective response against pathogens like HIV.

"In infectious disease, you want to find the best way to optimize the [initial] immune response, but in cancer you have an existing disease and you're looking to harness the immune system" to combat a self antigen, said Urdal. 


\section{ANALYSIS}

According to Urdal, the strong T cell responses elicited by Seder's methods are encouraging, but he cautioned that, at least in the PNAS study, another branch of the immune system-the B cell-based antibody response-appeared to be somewhat lacking.

"You want to activate as many arms of the immune system as you can," said Urdal. In Seder's study "they had great cellular response and lots of antibodies, but the antibodies had lower avidity" for HIV targets compared with what was seen in control monkeys.

Seder countered that the high level of $\mathrm{T}$ cell activity elicited by his vaccination methods makes it worthwhile to test the technique in tumor models irrespective of the antibody response.

Indeed, Celldex is pursuing the targeted DC approach in the clinic.

Celldex's CDX-1401, a fusion of the DEC-205-targeting mAb and cancer/testis antigen $1 \mathrm{~B}$ (CTAG1B; NY-ESO-1), a tumor antigen found in several cancers including melanoma, is in Phase I/II trials to treat NY-ESO-1-positive tumors.

Celldex also is providing material and technical support for Steinman's HIV trial.

Neither Celldex's cancer trial nor Steinman's HIV trial of the DEC205-targeting approach use the viral heterologous prime-boost method described in the PNAS study.

Celldex's SVP, CSO and founder Tibor Keler did say the company chose the DEC-205-targeting approach rather than the approach described in JCI because it is more straightforward to manufacture and deliver a mAbbased fusion protein than to formulate a protein aggregate.

"On the development side, there may be challenges in controlling the aggregation-based delivery compared with an antibody," added Keler, who was a coauthor on the PNAS paper.

Keler said it remains to be seen what combination of adjuvants, antigens and delivery techniques will prove most effective, so the next step is to combine the various reagents and techniques outlined in Seder's studies in further preclinical studies. Some of this work is already underway at Celldex.

Osherovich, L. SciBX 4(17); doi:10.1038/scibx.2011.474

Published online April 28, 2011

\section{REFERENCES}

1. Flynn, B.J. et al. Proc. Natl. Acad. Sci. USA; published online April 5, 2011; doi:10.1073/pnas.1103869108

Contact: Robert A. Seder, National Institute of Allergy and Infectious Diseases, National Institutes of Health, Bethesda, Md. e-mail: rseder@mail.nih.gov

Contact: Ralph M. Steinman, The Rockefeller University, New York, N.Y. e-mail: steinma@mail.rockefeller.edu

2. Kastenmüller, K. et al. J. Clin. Invest.; published online April 11, 2011; doi:10.1172/JCl45416

Contact: Robert A. Seder, National Institute of Allergy and Infectious Diseases, National Institutes of Health, Bethesda, Md. e-mail: rseder@mail.nih.gov

3. Nchinda, G. et al. J. Clin. Invest. 118, 1427-1436 (2008)

\section{COMPANIES AND INSTITUTIONS MENTIONED}

3M Co. (NYSE:MMM), St. Paul, Minn.

Celldex Therapeutics Inc. (NASDAQ:CLDX), Needham, Mass. Dendreon Corp. (NASDAQ:DNDN), Seattle, Wash.

Dynavax Technologies Corp. (NASDAQ:DVAX), Berkeley, Calif. National Institute of Allergy and Infectious Diseases,

Bethesda, Md.

The Rockefeller University, New York, N.Y. 